\title{
LINS NETO'S EXAMPLES OF FOLIATIONS AND THE MORI CONE OF BLOW-UPS OF $\mathbb{P}^{2}$
}

\author{
F. MONSERRAT
}

\begin{abstract}
We use a family of algebraic foliations given by A. Lins Neto to provide new evidences to a conjecture, related to the Harbourne-Hirschowitz's one and implying the Nagata's conjecture, which concerns the structure of the Mori cone of blow-ups of $\mathbb{P}^{2}$ at very general points.
\end{abstract}

\section{INTRODUCTION}

The Harbourne-Hirschowitz conjecture predicts the solution to the problem of determining the dimension of every linear system of curves of $\mathbb{P}^{2}$ (the projective plane over an algebraically closed field, which we shall assume to be $\mathbb{C}$ ) with assigned multiplicities at general points. This conjecture goes back to Segre [33] and it has been reformulated by several authors [17, 15, 22. We are interested in the following weaker form [19]:

Conjecture 1. Let $X$ be the blow-up of $\mathbb{P}^{2}$ at a finite set of points in very general position. Then, every integral curve $C$ on $X$ with negative self-intersection is a (-1)-curve of $X$ (that is, smooth, rational and such that $C^{2}=-1$ ).

Recall that a property is satisfied for $n$ closed points of $\mathbb{P}^{2}$ in very general position if it holds for all $n$-uples $\left(p_{1}, p_{2}, \ldots, p_{n}\right)$ belonging to the complement of a countable union of proper closed subvarieties of $\left(\mathbb{P}^{2}\right)^{n}$.

It is known that the statement of Conjecture 1 is satisfied by the rational curves and also by any curve whose image on $\mathbb{P}^{2}$ has a singularity of multiplicity 2 at one of the centers of the blow-up [6].

There are several results giving evidence to the Harbourne-Hirschowitz conjecture ([1], [20], [4], [5], [10], 35], 8] and [28] among many others). In the same spirit, the objective of this note will be to provide evidences to Conjecture 1. To do that, we shall consider other equivalent formulation (Conjecture 2) that predicts the structure of the closure of the Mori cone, $\overline{N E}(X)$, associated with a blow-up $X$ of $\mathbb{P}^{2}$ at very general points. In a more precise form, it states that any generator of extremal ray of $\overline{N E}(X)$ having non-negative intersection product with the canonical class $K_{X}$ of $X$ has null self-intersection. It is known that, when the number $n$ of blown-up points is greater than 1, the extremal rays of $\overline{N E}(X)$ in the half-space $\left(K_{X} \cdot z<0\right)$ are exactly those generated by the classes of the $(-1)$-curves and when $n \geq 9$ there are infinitely many of them 30 . When $n \leq 9$ the conjecture is true (see Remark 1) but very little is known on the intersection $\overline{N E}(X) \cap\left(K_{X} \cdot z \geq 0\right)$ when $n>9$.

An interesting aspect is that Conjecture 11 implies Nagata's conjecture [29] and, therefore, there is a connection with the symplectic packing problem in dimension four [27].

2000 Mathematics Subject Classification. 14C20; 14J25; 32S65.

Supported by Spain Ministry of Education MTM2007-64704. 
In section 4, we show an explicit family of smooth rational projective surfaces $X$ satisfying that the set of faces of the cone $N E(X)$ meeting the region $\left(K_{X} \cdot z=0\right)$ (resp., $\left(K_{X} \cdot z>0\right)$ ) is not finite (see Proposition 2). Under the assumption that $X$ is the blow-up of $\mathbb{P}^{2}$ at a set of $n \geq 12$ (resp., $n \geq 37$ ) points in very general position, we prove, also in Section 4, a behavior of the cone $N E(X)$ which agree with Conjecture 2, More specifically, on the one hand Theorem 1 (see also Remark 5) shows the existence of infinitely many rays which are contained in the boundary of $\overline{N E}(X)$ and are generated by elements in the region $\left(K_{X} \cdot z=0\right)$ (resp., $\left(K_{X} \cdot z>0\right)$ ) with null self-intersection; on the other hand Theorem 2 shows that, when $n \geq 37$, the set of the above mentioned rays which are in $\partial \overline{N E}(X) \cap\left(K_{X} \cdot z>0\right)$ correspond to infinitely many orbits of the action of the Cremona group. The proofs of these theorems use the examples of one-parametric families of algebraically integrable plane foliations provided by Lins Neto in [26] in relation with the so-called Poincaré and Painlevé problems. For this reason we devote Section 3 to summarize the necessary background on foliations, to state the results of [26] that we shall use and to prove a key fact in our development (Proposition 1). In Section 2 we briefly summarize basic definitions and facts on the Mori cone and we use them to state Conjecture 2,

We notice that, although the Lins Neto's families of foliations provide negative answers with respect to the Poincaré and Painlevé problems, in this paper they have revealed to be useful to show a positive result with respect to the Harbourne-Hirschowitz conjecture.

\section{The Mori COne And The CONJECTURE}

Let $X$ be a smooth projective surface and let $A(X):=(\operatorname{Pic}(X) / \equiv) \otimes \mathbb{R}$, where $\equiv$ denotes numerical equivalence. $A(X)$ is a real vector space whose dimension is $\rho(X):=\operatorname{rk} \operatorname{Pic}(X)$. We shall assume that $\rho(X) \geq 3$. The Mori cone of $X$ (also called Kleiman-Mori cone or cone of curves), which we shall denote by $N E(X)$, is defined to be the convex cone of $A(X)$ generated by the images of the effective classes in $\operatorname{Pic}(X)$; its closure with respect to the real topology will be denoted by $\overline{N E}(X)$. The $\mathbb{Z}$-bilinear form $\operatorname{Pic}(X) \times \operatorname{Pic}(X) \rightarrow \mathbb{Z}$ given by Intersection Theory induces a non-degenerated $\mathbb{R}$-bilinear pairing $A(X) \times A(X) \rightarrow \mathbb{R}$. For each pair $(x, y) \in A(X) \times A(X), x \cdot y$ will denote its image by this bilinear form, for each divisor $D$ on $X$, $[D]$ will be its image in $A(X)$ and, for each real number $\alpha$, $[D]_{>\alpha}$ (resp., $[D]_{\geq \alpha},[D]_{<\alpha},[D]_{\leq \alpha},[D]^{\perp}$ ) will denote the set of those $x \in A(X)$ such that $[D] \cdot x>\alpha$ (resp., $\geq \alpha,<\alpha, \leq \alpha,=0$ ).

Recall that, if $C$ is a convex cone of $A(X)$, a face of $C$ is a sub-cone $F \subseteq C$ such that $a+b \in F$ implies that $a, b \in F$, for all pair of elements $a, b \in C$. The 1-dimensional faces of $C$ are also called extremal rays of $C$.

Fix an ample divisor $H$ on $X$. By Kleiman's ampleness criterion, $[H] \cdot x>0$ for all $x \in \overline{N E}(X) \backslash\{0\}$ and, hence, the cone $\overline{N E}(X)$ is strongly convex. This implies that it is generated by its extremal rays. Consider the cone

$$
Q(X)=\left\{x \in A(X) \mid x^{2} \geq 0,[H] \cdot x \geq 0\right\} .
$$

One has that $Q(X) \subseteq \overline{N E}(X)$ [25, II.4.12.1] and, therefore, the extremal rays of $\overline{N E}(X)$ must be spanned by elements $x \in A(X)$ such that $x^{2} \leq 0$. Moreover, the extremal rays of $\overline{N E}(X)$ which are not in $Q(X)$ are spanned by classes of integral curves $C$ with $C^{2}<0$ [25, II.4.12.3]. As a consequence of the Mori cone theorem (see [25, III.1] for instance) the extremal rays of $\overline{N E}(X)$ meeting the region $\left[K_{X}\right]_{<0}$ are exactly those spanned by the images in $A(X)$ of the $(-1)$-curves. Moreover, if $C$ is an integral curve on $X$ such that $C^{2}<0$ then $[C]$ generates an extremal ray of $\overline{N E}(X)$ [25, II.4.12.2]. These considerations 
allow us to state the following equivalent formulation of Conjecture1(which holds trivially when $\rho(X)<3)$ :

Conjecture 2. If $X$ is the blow-up of $\mathbb{P}^{2}$ at a finite set of points in very general position then the extremal rays of the cone $\overline{N E}(X)$ in the region $\left[K_{X}\right]_{\geq 0}$ are contained in $\partial Q(X)$.

Remark 1. Notice that Conjecture 2 is true when $\rho(X) \leq 10$; in fact, the equality $\overline{N E}(X) \cap\left[K_{X}\right]_{\geq 0}=Q(X) \cap\left[K_{X}\right]_{\geq 0}$ holds in this case. Indeed, if $\rho(X) \leq 9$ then $Q(X) \cap$ $\left[K_{X}\right]_{\geq 0}=\overline{N E}(X) \cap\left[K_{X}\right]_{\geq 0}=\{0\}$ because $-K_{X}$ is ample. If $\rho(X)=10$ then one has that $\overline{N E}(X) \cap\left[K_{X}\right]_{\geq 0} \subseteq\left[K_{X}\right]^{\perp} \cap Q(X)=Q(X) \cap\left[K_{X}\right]_{\geq 0}$, where the inclusion holds because $-K_{X}$ is nef and there are not integral curves with negative self-intersection whose images belong to $\left[K_{X}\right]^{\perp}$, and the equality follows from the proof of [11, Cor. 1.ii].

Remark 2. Conjecture 1 is not equivalent to the fact that the equality $\overline{N E}(X) \cap\left[K_{X}\right]_{\geq 0}=$ $Q(X) \cap\left[K_{X}\right]_{\geq 0}$ holds for whichever surface $X$ obtained by blowing-up $\mathbb{P}^{2}$ at a finite set of points in very general position. Although a reformulation of Conjecture 1 in these terms is given in [6], its author (in a private communication to me and in a note which will appear elsewhere) asserts that this reformulation is only correct when $\rho(X) \leq 11$; in fact, he shows that the inclussion $Q(X) \cap\left[K_{X}\right]_{\geq 0} \subseteq \overline{N E}(X) \cap\left[K_{X}\right]_{\geq 0}$ is strict whenever $\rho(X)>11$ (independently of any conjecture).

\section{FAMilies OF ALGEBRAiCALLY INTEGRABLE FOLIATIONS}

With the exceptions of Lemma 1 and Proposition 1, this section is expository and its aim is, on the one hand, to summarize some basics facts concerning foliations (see [3] and [16]) and, on the other hand, to state the results of [26] that we shall use to obtain the main results of the paper.

Let $X$ be a smooth projective surface defined over $\mathbb{C}$ and let $\Theta_{X}$ be its associated tangent sheaf. An algebraic foliation with singularities (foliation, in the sequel) $\mathcal{F}$ on $X$ is given by an open covering $\left\{U_{j}\right\}_{j \in J}$ of $X$ and vector fields $v_{j} \in H^{0}\left(U_{j}, \Theta_{X}\right)$ with isolated zeroes such that $v_{i}=g_{i j} v_{j}$ on $U_{i} \cap U_{j}$, where $g_{i j} \in H^{0}\left(U_{i} \cap U_{j}, \mathcal{O}_{X}^{*}\right)$ for all $i, j \in J$. A closed point $p \in X$ is a singular point of $\mathcal{F}$ if it is a zero of $v_{j}$ for some $j \in J$. Notice that the set of singular points is finite. Given $p \in X$, a separatrix of $\mathcal{F}$ at $p$ will be an irreducible germ $f \in \mathcal{O}_{X, p}^{\text {hol }}$ (where $\mathcal{O}_{X}^{\text {hol }}$ denotes the sheaf of holomorphic functions) such that $v_{j}(f)$ is a multiple of $f$, if $p \in U_{j}$. An algebraic invariant curve $C$ will be an integral curve on $X$ such that the irreducible components of its germ at each point $p \in C$ (viewed as an element of $\mathcal{O}_{X, p}^{\text {hol }}$ ) are separatrices at $p$.

If $p$ is a singular point of $\mathcal{F}$ and $p \in U_{j}$, we shall say that $p$ is a non-degenerated singularity if the jacobian matrix $D v_{j}(p)$ is non-singular. In this case, if $\lambda_{1}$ and $\lambda_{2}$ denote the eigenvalues of $D v_{j}(p)$, the quotients $\lambda_{1} / \lambda_{2}$ and $\lambda_{2} / \lambda_{1}$ are called characteristic numbers of the singularity and they are analytic invariants. A singular point $p$ is called a reduced (resp., dicritical) singularity if their characteristic numbers are not positive rational numbers (resp., there exist infinitely many separatrices passing thorough $p$ ). If $p$ is a singularity of $\mathcal{F}$ such that the separatrices of $\mathcal{F}$ at $p$ are given by the levels of a meromorphic function of the type $\frac{u^{a}}{v^{b}}$ for certain local analytic coordinates $(u, v)$, then we say that $\mathcal{F}$ has a local meromorphic first integral at $p$ of the type $\frac{u^{a}}{v^{b}}$.

Given a vector field $p(x, y) \frac{\partial}{\partial x}+q(x, y) \frac{\partial}{\partial y}$, where $p$ and $q$ are polynomials on $\mathbb{C}^{2}$, it can be extended to a unique foliation $\mathcal{F}$ of $\mathbb{P}^{2}$. The singular points of $\mathcal{F}$ in the affine chart $\mathbb{C}^{2}$ are the common zeroes of $p$ and $q$. Moreover, there exists a positive integer $d$ such that 
the above vector field can be written in the form

$$
a(x, y) \frac{\partial}{\partial x}+b(x, y) \frac{\partial}{\partial y}+g(x, y)\left(x \frac{\partial}{\partial x}+y \frac{\partial}{\partial y}\right)
$$

where, either $a, b$ are polynomials of degree at most $d$ and $g$ is a homogeneous polynomial of degree $d$, or $g \equiv 0, \max \{\operatorname{deg}(a), \operatorname{deg}(b)\}=d$ and the homogeneous parts of $a$ and $b$ of degree $d$ are not of the form $x \cdot h$ and $y \cdot h$ respectively. The integer $d$ is the degree of $\mathcal{F}$, and it is also the number of tangencies of $\mathcal{F}$ with a generic line, linearly embedded in $\mathbb{P}^{2}$.

A foliation $\mathcal{F}$ of $\mathbb{P}^{2}$ has a rational first integral (or it is algebraically integrable) if there exists a rational map $R: \mathbb{P}^{2} \cdots \rightarrow \mathbb{P}^{1}$ such that the irreducible components of the closures of its fibers are algebraic invariant curves of $\mathcal{F}$. Taking homogeneous coordinates $[X, Y, Z]$ on $\mathbb{P}^{2}$, the map $R$ is defined by two homogeneous polynomials $F, G \in \mathbb{C}[X, Y, Z]$ of the same degree $m$ which can be taken in such a way that general fibers of $R$ are irreducible; the degree of the first integral $R$ is defined to be $m$. Hence, the foliation $\mathcal{F}$ determines a unique irreducible pencil (i.e., with irreducible general fibers) of plane curves $\mathcal{P}_{\mathcal{F}}:=$ $\langle F, G\rangle \subseteq H^{0}\left(\mathbb{P}^{2}, \mathcal{O}_{\mathbb{P}^{2}}(d)\right)$ given by the levels of the rational function $R=\frac{F}{G}$; moreover, the integral components of the curves in $\mathcal{P}_{\mathcal{F}}$ are exactly all the algebraic invariant curves.

A configuration over $\mathbb{P}^{2}$ will be a finite sequence $\mathcal{K}=\left(p_{1}, p_{2}, \ldots, p_{n}\right)$ of closed points such that $p_{1}$ belongs to $X_{1}:=\mathbb{P}^{2}$ and, inductively, if $i \geq 1$ then $p_{i}$ belongs to the blow-up $X_{i}$ of $X_{i-1}$ at $p_{i-1}$. Also, we shall denote by $\pi_{\mathcal{K}}: Z_{\mathcal{K}} \rightarrow \mathbb{P}^{2}$ the morphism given by the composition of all the successive blow-ups centered at the points of $\mathcal{K}$.

Consider a non-degenerated foliation $\mathcal{F}$ (that is, a foliation whose singularities are nondegenerated) of $\mathbb{P}^{2}$. Seidenberg's result of reduction of singularities 34] proves the existence of a sequence of blow-ups $X_{n+1} \stackrel{\pi_{n}}{\longrightarrow} X_{n} \stackrel{\pi_{n-1}}{\longrightarrow} \cdots \stackrel{\pi_{2}}{\longrightarrow} X_{2} \stackrel{\pi_{1}}{\longrightarrow} X_{1}:=\mathbb{P}^{2}$ and foliations $\mathcal{F}_{i}$ on $X_{i}\left(\mathcal{F}_{1}=\mathcal{F}\right.$ and the remaining ones are successive transforms of $\left.\mathcal{F}\right)$ such that $\mathcal{F}_{n+1}$ has only reduced singularities. If, in addition, $\mathcal{F}$ has a rational first integral, $R$, then the non-reduced singularities are exactly the dicritical ones; moreover, elementary calculations using the local equations of the blow-up show that the Seidenberg's reduction process coincides with the minimal composition of point blow-ups $\pi_{\mathcal{F}}: Z_{\mathcal{F}} \rightarrow \mathbb{P}^{2}$ eliminating the indeterminacies of $R$. The morphism $\pi_{\mathcal{F}}$ and the configuration given by the sequence of centers of the blow-ups used to get it, which we shall denote by $\mathcal{B}_{\mathcal{F}}$, are essentially unique because different admissible (in the obvious way) arrangements of the points give rise to $\mathbb{P}^{2}$-isomorphic surfaces. $\mathcal{B}_{\mathcal{F}}$ is called configuration of base points of $\mathcal{P}_{\mathcal{F}}$.

Definition 1. A one-parametric family of foliations of $\mathbb{P}^{2}$ will be a set $\left\{\mathcal{F}_{\alpha}\right\}_{\alpha \in U}$, where $U$ is a connected open subset of $\mathbb{C}$ and $\mathcal{F}_{\alpha}$ are foliations of $\mathbb{P}^{2}$ which extend polynomial vector fields $a_{\alpha}(x, y) \frac{\partial}{\partial x}+b_{\alpha}(x, y) \frac{\partial}{\partial y}$ on $\mathbb{C}^{2}$ such that the coefficients of $a_{\alpha}(x, y)$ and $b_{\alpha}(x, y)$ are functions of $\alpha$ which are holomorphic in $U$.

In [26], Lins Neto defines, for all integers $d \geq 2$, a one-parametric familiy of foliations of degree $d, \Upsilon^{d}=\left\{\mathcal{F}_{\alpha}^{d}\right\}_{\alpha \in \mathbb{C} \backslash A^{d}}$, where $A^{d}$ is a finite subset of $\mathbb{C}$. For all $d \geq 2$, the family $\Upsilon^{d}$ has non-degenerated singularities of fixed analytic type [26, Def. 1] and all the foliations in the family have the same dicritical singularities. Moreover, there exists a dense countable subset $E^{d} \subseteq \mathbb{C} \backslash A^{d}$ such that $\mathcal{F}_{\alpha}^{d}$ has a rational first integral for all $\alpha \in E^{d}$. If $d \in\{2,3,4\}$ and $\alpha \in E^{d}$, then the general algebraic invariant curves of $\mathcal{F}_{\alpha}^{d}$ are elliptic curves. If $d \geq 5$, then the following property is satisfied: for any $k>0$ the set $\left\{\alpha \in E^{d} \mid\right.$ the genus of a general algebraic invariant curve by $\mathcal{F}_{\alpha}^{d}$ is $\left.\leq k\right\}$ is finite. We point out here that, althouth the existence of the sets $E^{d}$ is proved, they are not explicitly described in [26]. Next, we shall summarize other properties of these families that will be of interest for us (see [26] for complete details). 
With respect to the family $\Upsilon^{4}$, there are 9 lines which are common invariant curves of all the foliations of the family and the dicritical singularities are the 12 points of intersection among these lines (see [26, Fig. 1]). Moreover, they are of radial type, that is, they have a local meromorphic first integral of the type $u / v$ for certain local coordinates $(u, v)$.

The family $\Upsilon^{3}=\left\{\mathcal{F}_{\alpha}^{3}\right\}_{\alpha \in \mathbb{C} \backslash\left\{0,1, j, j^{2}\right\}}$ is such that $\mathcal{F}_{\alpha}^{3}=\mathcal{G}_{\alpha}$, where $\mathcal{G}_{\alpha}$ is the foliation satisfying the following property: $\mathcal{F}_{\alpha}^{4}$ is the pull-back of $\mathcal{G}_{\alpha}$ by the map $T(x, y)=(x+y, x \cdot y)$ [26. Sect. 2.3]. The foliations in $\Upsilon^{3}$ have 5 common invariant curves: 2 conics and 3 lines. The 8 dicritical singularities are the points of intersection among theses curves [26, Fig. 4]. Three of them are of radial type and the remaining ones have a meromorphic first integral of the type $u^{2} / v$.

The foliations in $\Upsilon^{2}$ are obtained from those in $\Upsilon^{3}$ by a Cremona transformation [26, Sect. 2.4]. All of them have 2 common invariant curves: a quartic $Q$ and a line $R$. There are 5 dicritical singularities. Two of them (say $M$ and $N$ ) are smooth points of $Q$ and are points of tangency of $Q$ and $R$; they have a meromorhic first integral of the type $u^{2} / v$. The remaining ones (say $J, K$ and $L$ ) are cuspidal points of $Q$ (see [26, Fig. 7]) and have a meromorphic first integral of the type $u^{3} / v^{2}$.

The foliations in $\Upsilon^{3 r-1}$ (with $r \geq 2$ ) are obtained by pulling-back those in $\Upsilon^{2}$ by certain rational maps $F: \mathbb{P}^{2} \rightarrow \mathbb{P}^{2}$ (see [26, Sect. 3.1]). The dicritical singularities are those in $F^{-1}(\{J, K, L, M, N\}) . J, K$ and $L$ are not critical values of $F$ and $F^{-1}(\{J, K, L\})$ contains $3 r^{2}$ singularities with meromorphic first integral of the type $u^{3} / v^{2} . M$ and $N$ are critical values of $F$ and $F^{-1}(\{M, N\})$ contains $2 r$ singularities with meromorphic first integral of the type $u^{r} / v^{2}$.

In the same way as above, the foliations in $\Upsilon^{3 r}$ (with $r \geq 2$ ) are obtained by pullingback those in the family $\Upsilon^{3}$ by certain rational maps $\mathbb{P}^{2} \rightarrow \mathbb{P}^{2}$ (see [26, Sect. 3.2]). The dicritical singularities are the pre-images of the dicritical singularities of the foliations in $\Upsilon^{3}$. The analytic types of these singularities are the following ones: one radial singularity, $3 r^{2}$ singularities with local meromorphic first integral of the type $u^{2} / v, 2 r$ singularities with local meromorphic first integral of the type $u^{r} / v$ and $2 r$ more with meromorphic first integral of the type $u^{r} / v^{2}$.

The foliations in $\Upsilon^{3 r+1}$ (with $r \geq 2$ ) are obtained also by pulling-back those in $\Upsilon^{4}$ by certain rational maps $\mathbb{P}^{2} \rightarrow \mathbb{P}^{2}$. The dicritical singularities are the pre-images of those of the foliations in $\Upsilon^{4}$ and they have the following analytic types: $3 r^{2}+3$ radial singularities and $6 r$ singularities with local meromorphic first integral of the type $u^{r} / v$.

Remark 3. Although all the above foliations $\mathcal{F}_{\alpha}^{d}$ have local meromorphic first integrals at their dicritical singular points, only those corresponding to indices $\alpha$ in $E^{d}$ have a rational first integral. We notice that, for $\alpha \in E^{d}$, the knowledge of the type of the local meromorphic first integrals of $\mathcal{F}_{\alpha}^{d}$ is far to provide sufficient information to recover the rational first integral of $\mathcal{F}_{\alpha}^{d}$, even the singularity types of the generic algebraic invariant curves at the dicritical singular points. Indeed, if $\mathcal{F}_{\alpha}^{d}$ (with $\alpha \in E^{d}$ ) has a local meromorphic first integral of the type $u^{\rho} / v^{\delta}$ (with $\rho$ and $\delta$ relatively primes) at a dicritical point $p$ we are saying that the germs at $p$ of the curves of the pencil $\mathcal{P}_{\mathcal{F}_{\alpha}^{d}}$ are $s\left(\lambda_{1} P\left(u^{\rho}, v^{\delta}\right)+\lambda_{2} Q\left(u^{\rho}, v^{\delta}\right)\right)$, $\left(\lambda_{1}, \lambda_{2}\right) \in \mathbb{C}^{2} \backslash\{(0,0)\}$, where $s$ is a unit of $\mathcal{O}_{\mathbb{P}^{2}, p}^{h o l}$ and $P$ and $Q$ are homogeneous polynomials of the same degree, say $k_{p}$ (see [14, Section 2.9] for instance). To determine the singularity types of the generic curves of the pencil $\mathcal{P}_{\mathcal{F}_{\alpha}^{d}}$ one needs to know the set of values $k_{p}$, which is essentially an equivalent datum to the degree of the rational first integral (see [13, Th. 3.7]). Poincaré considered, in [31, 32, the following classical problem: to obtain a bound of such a degree in terms of the degree of the foliation. It is well-known that, in 
general, it is not possible to find that bound (even if the analytic types of the singularities of the foliation are given, as Lins Neto proves in [26] using the mentioned families).

Now, we shall see that all the foliations of a given one-parametric family $\Upsilon^{d}$ have a common resolution of singularities. For this purpose, we shall prove the following

Lemma 1. Let $\left\{\mathcal{F}_{\alpha}\right\}_{\alpha \in U}$ be a one-parametric family of foliations of $\mathbb{P}^{2}, U$ being a connected open subset of $\mathbb{C}$. Let $p$ be a point of $\mathbb{P}^{2}$. Suppose that:

(a) All the foliations $\mathcal{F}_{\alpha}$ have a non-degenerated singularity at $p$ of the same analytic type.

(b) The characteristic numbers of $\mathcal{F}_{\alpha}, \alpha \in U$, at $p$ are rational and positive, say $a / b$ and $b / a$, where $a, b \in \mathbb{N}$ are relatively primes.

(c) All the foliations $\mathcal{F}_{\alpha}$ have two common separatrices through $p$.

Then, the configuration of infinitely near points involved in the resolution of the singularity of $\mathcal{F}_{\alpha}$ at $p$ is constant for all $\alpha \in U$ (that is, the singularities at $p$ of all the foliations in the family have a common resolution).

Proof. Let $\mathcal{C}_{\alpha}=\left(p_{1}(\alpha)=p, p_{2}(\alpha) \ldots, p_{s}(\alpha)\right), \alpha \in U$, be the configuration of those infinitely near points involved in the resolution of the singularity of $\mathcal{F}_{\alpha}$ at $p$. The result is trivial when $s=1$, that is, the singularity is of radial type. So, we shall assume that either $a$ or $b$ is greater than 1 .

Applying [26, Lem. 1] one has that, for each $\alpha \in U$, there exists a holomorphic coordinate system $\left(W_{\alpha},\left(u_{\alpha}, v_{\alpha}\right)\right)$ with $p \in W_{\alpha}, u_{\alpha}(p)=v_{\alpha}(p)=0$, such that $\frac{u_{\alpha}^{a}}{v_{\alpha}^{b}}$ is a meromorphic first integral of $\mathcal{F}_{\alpha}$ in a neighborhood of $p$. Therefore, for each $\alpha \in U$, the local analytic separatrices of $\mathcal{F}_{\alpha}$ through $p$ are the irreducible components of the analytic germs in the local linear pencil $\Delta_{\alpha}:=\left\{\lambda u_{\alpha}^{a}+\mu v_{\alpha}^{b}=0 \mid(\lambda, \mu) \in \mathbb{C}^{2} \backslash\{(0,0)\}\right\}$. Moreover, since $\mathcal{C}_{\alpha}$ is also the configuration of base points of $\Delta_{\alpha}$, it is clear that $p_{j}(\alpha)$ belongs to the exceptional divisor created by blowing-up $p_{j-1}(\alpha)$ (for all $j \geq 2$ ) and that, in order to prove the equalities $\mathcal{C}_{\alpha}=\mathcal{C}_{\beta}$ for all $\alpha, \beta \in U$, it suffices to show that all the points $p_{2}(\alpha)$ coincide. Now, notice that all the germs in $\Delta_{\alpha}$ are irreducible and have the same tangent direction, except one of them (defined by $v_{\alpha}^{b}=0$ if the inequality $a<b$ is assumed). Hence, the strict transform in the blow-up at $p$ of one of the two common separatrices given in (c) must pass through $p_{2}(\alpha)$. Therefore there are, at most, two possibilities (which do not depend on the value of $\alpha$ ) for the point $p_{2}(\alpha)$, say $e_{1}$ and $e_{2}$, given by the tangent directions defined by the common separatrices. If $e_{1}=e_{2}$, then the result follows. So, we shall assume that $e_{1}$ and $e_{2}$ are different points of the first exceptional divisor $E$. Then, for each $\alpha \in U$, $p_{2}(\alpha)$ coincides with one of these points, and the remaining one is a reduced singularity of the transform of $\mathcal{F}_{\alpha}$.

Observe that, on the one hand, for each $i=1,2$, there exists a holomorphic function $f_{i}$ on $U$ such that the characteristic numbers of the transform of the foliation $\mathcal{F}_{\alpha}$ at the point $e_{i}$ are $f_{i}(\alpha)$ and $1 / f_{i}(\alpha)$ (both functions $f_{i}$ and $1 / f_{i}$ are defined in $U$ because we are assuming that the singularities are non-degenerated). On the other hand, for each $\alpha \in U$, $p_{2}(\alpha)$ is a dicritical singularity of the transform of $\mathcal{F}_{\alpha}$ and its characteristic numbers are $\frac{a}{b-a}$ and $\frac{b-a}{a}$ (assuming that $a<b$ ). Now, consider the following holomorphic functions defined in $U: g_{i}(\alpha):=\left(f_{i}(\alpha)-\frac{a}{b-a}\right) \cdot\left(f_{i}(\alpha)-\frac{b-a}{a}\right), \quad i=1,2$. Notice that $g_{1}(\alpha) g_{2}(\alpha)=0$ and $g_{1}(\alpha)-g_{2}(\alpha) \neq 0$ for all $\alpha \in U$. Since both functions are holomorphic, it follows that one of them (say $g_{i_{0}}$ ) is identically zero in $U$. Therefore $p_{2}(\alpha)=e_{i_{0}}$ for all $\alpha \in U$.

Proposition 1. Let $d \geq 2$ be an integer. Then, the configurations $\mathcal{B}_{\mathcal{F}_{\alpha}^{d}}$ coincide (up to re-arrangement) for all $\alpha \in \mathbb{C} \backslash A^{d}$. 
Proof. Taking into account the above description of the dicritical singularities of the foliations in the families $\Upsilon^{d}$ and applying Lemma 1, the result is easily deduced when $d \not \equiv 2$ mod 3. In the case of $\Upsilon^{2}$, it can be checked by inspection that the infinitely near points involved in the resolution of the singularities at $J, K$ and $L$ are the same for all the foliations in the family. By Lemma 1, the same is true for $M$ and $N$. Therefore, $\mathcal{B}_{\mathcal{F}_{\alpha}^{2}}$ does not depend on $\alpha$. In the case of $\Upsilon^{3 r-1}$ (for $r \geq 2$ ) the $3 r^{2}$ singularities in $F^{-1}(\{J, K, L\})$ have also the same resolution; the reason is the following one: for any $p \in\{J, K, L\}$ and for any $q \in F^{-1}(p), F$ defines a biholomorphism in a neighborhood of $q$, since $p$ is not a critical value of $F$. Again by Lemma1, the same occurs for the remaining singularities.

In view of the above proposition, we shall consider that, for a fixed $d \geq 2$, all the configurations $\mathcal{B}_{\mathcal{F}_{\alpha}^{d}}$ are the same and we shall denote it by $\mathcal{B}^{d}$. Also, $X^{d}$ will denote the surface $Z_{\mathcal{B}^{d}}$ obtained by blowing-up the points in $\mathcal{B}^{d}$.

\section{The Mori CONE OF THE BLOW-Ups}

The rest of the paper is devoted to prove the two main results. We shall use the notations of the preceding sections.

In order to state and prove the first of these results, we denote by $\Delta(C)$ the set of faces of a convex cone $C$ and, for each integer $d \geq 2$, we consider the function

$$
\Psi^{d}: E^{d} \rightarrow \Delta\left(N E\left(X^{d}\right)\right)
$$

which maps every $\alpha \in E^{d}$ to $\left[D_{d, \alpha}\right]^{\perp} \cap N E\left(X^{d}\right)$, where $D_{d, \alpha}$ denotes the strict transform on $X^{d}$ of a general invariant curve by $\mathcal{F}_{\alpha}^{d}$ that is, a general curve of the pencil $\mathcal{P}_{\mathcal{F}_{\alpha}^{d}}$ (note that all these foliations have a rational first integral). This map is well-defined. Indeed, since the complete linear system $\left|D_{d, \alpha}\right|$ is base-point-free, one has that $\left[D_{d, \alpha}\right]^{\perp}$ is a supporting hyperplane of the cone $N E\left(X^{d}\right)$ and, therefore, $\left[D_{d, \alpha}\right]^{\perp} \cap N E\left(X^{d}\right)$ is a face of $N E\left(X^{d}\right)$.

Proposition 2. For each positive integer $d \geq 2$, the map $\Psi^{d}$ is injective. Moreover:

(a) If $d \leq 4$, then $\Psi^{d}(\alpha) \cap\left[K_{X^{d}}\right]^{\perp} \neq\{0\}$ for all $\alpha \in E^{d}$.

(b) If $d \geq 5$, then for any $k>0$ the set $\left\{\alpha \in E^{d} \mid \Psi^{d}(\alpha) \subseteq\left[K_{X^{d}}\right]_{\leq k}\right\}$ is finite.

In particular, the set of faces of the cone $N E\left(X^{d}\right)$ meeting the region $\left[K_{X^{d}}\right]^{\perp}$ is not finite and, if $d \geq 5$, the same happens for the set of faces of $N E\left(X^{d}\right)$ meeting the region $\left[K_{X^{d}}\right]_{>0}$.

Proof. Set any integer $d \geq 2$. By applying Bézout's theorem to two general curves of $\mathcal{P}_{\mathcal{F}_{\alpha}^{d}}$, it is easy to deduce that, for each $\alpha \in E^{d}, D_{d, \alpha}^{2}=0$ and, therefore, $\left[D_{d, \alpha}\right]$ belongs to $\Psi^{d}(\alpha)$. Now, two different values $\alpha, \beta \in E^{d}$ give rise to two different faces $\Psi^{d}(\alpha)$ and $\Psi^{d}(\beta)$. Indeed, if the two faces were the same then both foliations $\mathcal{F}_{\alpha}^{d}$ and $\mathcal{F}_{\beta}^{d}$ would have the same invariant curves [12, Th. 1] and, hence, they would coincide; this is a contradiction. So, the map $\Psi^{d}$ is injective.

Clauses (a) and (b) follow easily taking into account the paragraph after Definition 1 and the Adjunction Formula applied to the divisors $D_{d, \alpha}$.

Remark 4. In Section 3, it is given the local analytic type of the first integrals of the foliations of each family at each dicritical singularity. This allows to compute, for each $d \geq 2$, the number of points $\ell\left(\mathcal{B}^{d}\right)$ involved in the configuration $\mathcal{B}^{d}$ (see Remark 3). In fact, one has that $\ell\left(\mathcal{B}^{2}\right)=13, \ell\left(\mathcal{B}^{3}\right)=13, \ell\left(\mathcal{B}^{4}\right)=12, \ell\left(\mathcal{B}^{3 n+1}\right)=9 n^{2}+3, \ell\left(\mathcal{B}^{3 n-1}\right)=$ $10 n^{2}+3\left(1-(-1)^{n}\right) n / 2$ and $\ell\left(\mathcal{B}^{3 n}\right)=9 n^{2}+1+3\left(1-(-1)^{n}\right) n / 2$ for all $n \geq 2$. 
Let $\mathcal{K}=\left(p_{1}, p_{2}, \ldots, p_{n}\right)$ be an arbitrary configuration over $\mathbb{P}^{2}$. Each blow-up at $p_{i}$ gives rise to an exceptional divisor $E_{i}$ whose total (resp., strict) transform on $Z_{\mathcal{K}}$ will be denoted by $E_{i}^{\mathcal{K}}$ (resp., $\tilde{E}_{i}^{\mathcal{K}}$ ). In the same way, for each effective divisor $C$ on $X, C^{\mathcal{K}}$ (resp., $\tilde{C}^{\mathcal{K}}$ ) will be the total (resp., strict) transform of $C$ on $Z_{\mathcal{K}}$. The system $\left\{\left[L^{\mathcal{K}}\right],\left[E_{0}^{\mathcal{K}}\right],\left[E_{1}^{\mathcal{K}}\right], \ldots,\left[E_{n}^{\mathcal{K}}\right]\right\}$ is a basis of the vector space $A\left(Z_{\mathcal{K}}\right), L$ denoting a general line on $\mathbb{P}^{2}$.

For each positive integer $n$, there exists a smooth projective variety $Y_{n-1}$ whose closed points are naturally identified with the configurations over $\mathbb{P}^{2}$ with $n$ points. These varieties, known as iterated blow-ups, were introduced by Kleiman in [23] and 24 and they have also been treated in [18. There is a family of smooth projective morphisms $Y_{n} \rightarrow Y_{n-1}$ and relative divisors $F_{0}, F_{1}, \ldots, F_{n}$ on $Y_{n}$ such that the fiber over a given configuration $\mathcal{K}=\left(p_{1}, \ldots, p_{n}\right)$ (viewed as a point of $\left.Y_{n-1}\right)$ is isomorphic to the surface $Z_{\mathcal{K}}$ obtained by blowing-up the points in $\mathcal{K}$ and, if $i \geq 1$ (resp., $i=0$ ), the restriction of $F_{i}$ to this fiber corresponds to the total transform $E_{i}^{\mathcal{K}}$ of the exceptional divisor appearing in the blow-up centered at $p_{i}$ (resp., the total transform of a general line of $\left.\mathbb{P}^{2}\right)[18$, Prop. I.2]. For each positive integer $d$ and for each sequence of non-negative integers $m_{1}, \ldots, m_{n}$ we apply the Semicontinuity Theorem [21, III.12.8] to the invertible sheaf $\mathcal{O}_{Y_{n}}\left(d F_{0}-m_{1} F_{1}-m_{2} F_{2}-\ldots-m_{n} F_{n}\right)$, obtaining that the function $Y_{n-1} \rightarrow \mathbb{Z}$ given by $\mathcal{K} \mapsto h^{0}\left(Z_{\mathcal{K}}, \mathcal{O}_{Z_{\mathcal{K}}}\left(d L^{\mathcal{K}}-\sum_{i} m_{i} E_{i}^{\mathcal{K}}\right)\right)$ is upper-semicontinuous. Moreover, the subset $U \subseteq Y_{n-1}$ given by the configurations $\left(p_{1}, p_{2}, \ldots, p_{n}\right)$ such that $p_{i} \in \mathbb{P}^{2}$ for all $i=1,2, \ldots, n$ is dense in $Y_{n}$ (see [23]). Then, denoting by $\mathcal{K}_{0}(n)$ a configuration whose elements are $n$ points of $\mathbb{P}^{2}$ in very general position, one has that

$$
h^{0}\left(Z_{\mathcal{K}}, \mathcal{O}_{Z_{\mathcal{K}}}\left(d L^{\mathcal{K}}-\sum_{i} m_{i} E_{i}^{\mathcal{K}}\right)\right) \geq h^{0}\left(Z_{\mathcal{K}_{0}(n)}, \mathcal{O}_{Z_{\mathcal{K}_{o}(n)}}\left(d L^{\mathcal{K}_{0}(n)}-\sum_{i} m_{i} E_{i}^{\mathcal{K}_{0}(n)}\right)\right)
$$

for all triplets $\left(d, \mathcal{K},\left\{m_{i}\right\}_{i=1}^{n}\right)$ such that $d$ is a positive integer, $\mathcal{K}$ is a configuration with $n$ points and $\left\{m_{i}\right\}_{i=1}^{n}$ is a sequence of non-negative integers.

Let $n$ be a positive integer and set $\mathcal{K}_{0}(n)=\left(p_{1}, p_{2}, \ldots, p_{n}\right)$. For each configuration $\mathcal{C}=\left(p_{1}^{\prime}, p_{2}^{\prime}, \ldots, p_{m}^{\prime}\right)$ such that $m \leq n$, the map $A\left(Z_{\mathcal{C}}\right) \rightarrow A\left(Z_{\mathcal{K}_{0}(n)}\right)$ given by $\left[L^{\mathcal{C}}\right] \mapsto$ $\left[L^{\mathcal{K}_{0}(n)}\right]$ and $\left[E_{i}^{\mathcal{C}}\right] \mapsto\left[E_{i}^{\mathcal{K}_{0}(n)}\right]$ for $i=1,2, \ldots, m$, is a monomorphism of vector spaces; then, identifying $A\left(Z_{\mathcal{C}}\right)$ with its image, we can assume an inclusion $A\left(Z_{\mathcal{C}}\right) \subseteq A\left(Z_{\mathcal{K}_{0}(n)}\right)$. We shall use this identification in the rest of the paper. Also, if $x=\lambda_{0}\left[L^{\mathcal{K}_{0}(n)}\right]+\sum_{i=1}^{n} \lambda_{i}\left[E_{i}^{\mathcal{K}_{0}(n)}\right] \in$ $A\left(Z_{\mathcal{K}_{0}(n)}\right)$, with $\lambda_{i} \in \mathbb{R}$ for all $i$, we shall denote by $N(x)$ the cardinality of the set $\left\{i \mid 1 \leq i \leq n\right.$ and $\left.\lambda_{i} \neq 0\right\}$.

Finally, we shall prove the two results (Theorems 1 and 2) that provide an evidence to Conjecture 2.

Theorem 1. Let $X_{n}$ be the surface $Z_{\mathcal{K}_{0}(n)}$ obtained by blowing-up $n \geq 12$ points of $\mathbb{P}^{2}$ in very general position. Consider the sets $S(n):=\left\{d \in \mathbb{Z} \mid d \geq 2\right.$ and $\left.\ell\left(\mathcal{B}^{d}\right) \leq n\right\}$ and $E(n):=\left\{(d, \alpha) \mid d \in S(n)\right.$ and $\left.\alpha \in E^{d}\right\}$.

(a) $\left[D_{d, \alpha}\right] \in \partial \overline{N E}\left(X_{n}\right) \cap \partial Q\left(X_{n}\right)$ for all $(d, \alpha) \in E(n)$.

(b) If $\left(d_{1}, \alpha_{1}\right),\left(d_{2}, \alpha_{2}\right) \in E(n)$, then $\mathbb{R}_{>0}\left[D_{d_{1}, \alpha_{1}}\right] \neq \mathbb{R}_{>0}\left[D_{d_{2}, \alpha_{2}}\right]$ whenever either $d_{1}=$ $d_{2}$ and $\alpha_{1} \neq \alpha_{2}$, or $d_{1} \neq d_{2}$ and $\left\{d_{1}, d_{2}\right\} \neq\{2,3\}$.

(c) If $d \in S(n) \cap\{2,3,4\}$, then $\left[D_{d, \alpha}\right] \in\left[K_{X_{n}}\right]^{\perp}$ for all $\alpha \in E^{d}$.

(d) For each $k>0$, the set $\left\{\alpha \in E^{d} \mid\left[D_{d, \alpha}\right] \in\left[K_{X_{n}}\right]_{\leq k}\right\}$ is finite whenever $d \in$ $S(n) \backslash\{2,3,4\}$.

In particular, for all $n \geq 12$ (resp., $\geq 37$ ), the set of rays contained in $\partial \overline{N E}\left(X_{n}\right) \cap$ $\partial Q\left(X_{n}\right) \cap\left[K_{X_{n}}\right]^{\perp}$ (resp., $\left.\partial \overline{N E}\left(X_{n}\right) \cap \partial Q\left(X_{n}\right) \cap\left[K_{X_{n}}\right]_{>0}\right)$ is not finite, and the cone $\overline{N E}\left(X_{n}\right)$ has infinitely many faces meeting the region $\left[K_{X_{n}}\right]^{\perp}$ (resp., $\left[K_{X_{n}}\right]_{>0}$ ). 
Proof. For each $d \in S(n)$ consider a configuration $\mathcal{C}^{d}:=\left(p_{1}, p_{2}, \ldots, p_{r}, q_{1}, q_{2}, \ldots, q_{n-r}\right)$, where $\mathcal{B}^{d}=\left(p_{1}, p_{2}, \ldots, p_{r}\right)$ and $q_{1}, q_{2}, \ldots, q_{n-r}$ are different closed points of $\mathbb{P}^{2}$ which are not in $\mathcal{B}^{d}$. For every pair $(d, \alpha) \in E(n)$ one has that $D_{d, \alpha}^{2}=0$ and, therefore, $\left[D_{d, \alpha}\right]$ belongs to the boundary of $Q\left(Z_{\mathcal{C}^{d}}\right)=Q\left(X_{n}\right)$; so, it also belongs to $\partial \overline{N E}\left(Z_{\mathcal{C}^{d}}\right)[25$, II.4.12.2]. By (11), $\overline{N E}\left(X_{n}\right) \subseteq \overline{N E}\left(Z_{\mathcal{C}^{d}}\right)$ and, since $\left[D_{d, \alpha}\right] \in Q\left(X_{n}\right) \subseteq \overline{N E}\left(X_{n}\right)$, one has that $\left[D_{d, \alpha}\right] \in \partial \overline{N E}\left(X_{n}\right)$. This proves (a).

Consider $d_{1}, d_{2} \in S(n)$ and $\alpha_{i} \in E^{d_{i}}, i=1,2$. If $d_{1}=d_{2}$ and $\alpha_{1} \neq \alpha_{2}$, one has clearly that $\left[D_{d_{1}, \alpha_{1}}\right]$ and $\left[D_{d_{1}, \alpha_{2}}\right]$ cannot be proportional since, in this case, $\Psi^{d_{1}}\left(\alpha_{1}\right)=\Psi^{d_{1}}\left(\alpha_{2}\right)$ but, by Proposition 2, the map $\Psi^{d_{1}}$ is injective. If $d_{1} \neq d_{2}$ and $\left\{d_{1}, d_{2}\right\} \neq\{2,3\}$ then $\ell\left(\mathcal{B}^{d_{1}}\right) \neq \ell\left(\mathcal{B}^{d_{2}}\right)$ (see Remark 4). But, since $N\left(\left[D_{d_{i}, \alpha_{i}}\right]\right)=\ell\left(\mathcal{B}^{d_{i}}\right), i=1,2$, (recall the notation introduced before the statement of the theorem) one has that $\left[D_{d_{1}, \alpha_{1}}\right]$ and $\left[D_{d_{2}, \alpha_{2}}\right]$ cannot be proportional. Therefore, (b) holds.

If $d \in S(n) \cap\{2,3,4\}$, taking into account the paragraph after Definition 1 and the Adjunction Formula, one has that $\left[K_{X_{n}}\right] \cdot\left[D_{d, \alpha}\right]=K_{X^{d}} \cdot D_{d, \alpha}=0$ for all $\alpha \in E^{d}$ (as in the proof of Proposition 2). This proves (c). Clause (d) follows in a similar manner.

In particular, from (a), (b) and (c) it follows that the set of rays contained in $\partial \overline{N E}\left(X_{n}\right) \cap$ $\partial Q\left(X_{n}\right) \cap\left[K_{X_{n}}\right]^{\perp}$ is not finite. Also, from (a), (b) and (d) it follows that, if $n \geq 37$, the same happens for the set of rays contained in $\partial \overline{N E}\left(X_{n}\right) \cap \partial Q\left(X_{n}\right) \cap\left[K_{X_{n}}\right]_{>0}$.

Finally, we shall prove the last assertion of the statement. By the Hodge index theorem [21, V.1.9], the index of the bilinear pairing $A\left(X_{n}\right) \times A\left(X_{n}\right) \rightarrow \mathbb{R}$ induced by the intersection product is $(1, n)$. Then, taking coordinates in a certain basis, $Q\left(X_{n}\right)$ can be seen as the half-cone over an Euclidean ball of dimension $n$, which is strictly convex. This fact and the inclusion $Q\left(X_{n}\right) \subseteq \overline{N E}\left(X_{n}\right)$ imply that two non-proportional classes of the form $\left[D_{d, \alpha}\right]$ must belong to different faces of the cone $\overline{N E}\left(X_{n}\right)$.

Remark 5. Although the statement of Theorem 1 shows that, for each integer $n \geq 37$, there exist infinitely many rays cutting the region $\partial \overline{N E}\left(X_{n}\right) \cap \partial Q\left(X_{n}\right) \cap\left[K_{X_{n}}\right]_{>0}$ (which are spanned by elements of the form $\left[D_{d, \alpha}\right]$, with $\left.(d, \alpha) \in E(n)\right)$, a stronger fact is implicit in that statement (recall also Remark (4): if $f_{n}$ denotes the maximum of the numbers $N\left(\left[D_{d, \alpha}\right]\right)$, with $(d, \alpha) \in E(n)$ (notice that they depend only on $d$ ), then $\lim _{n \rightarrow \infty} f_{n}=$ $+\infty$. This implies that, for each fixed positive integer $k$, there exists an integer $n_{0}>k$ such that, for each $n \geq n_{0}$, one can find infinitely many rays $\mathbb{R}_{>0} z$ cutting the region $\partial \overline{N E}\left(X_{n}\right) \cap \partial Q\left(X_{n}\right) \cap\left[K_{X_{n}}\right]_{>0}$ and such that the number of exceptional divisors $E$ on the blow-up $X_{n}$ with $z \cdot[E]>0$ is greater than $k$.

Now, we shall use the action of the Cremona group in order to extend further the result given in the above theorem. Let us recall briefly this action, referring the reader to [9] and [7] for more details.

Let $X_{n}$ be as in the statement of Theorem 1. Following preceding notations, consider $A\left(X_{n}\right)$ as the direct sum $\mathbb{Z}\left[L^{\mathcal{K}_{0}(n)}\right] \oplus \mathbb{Z}\left[E_{1}^{\mathcal{K}_{0}(n)}\right] \oplus \cdots \oplus \mathbb{Z}\left[E_{n}^{\mathcal{K}_{0}(n)}\right]$. $A\left(X_{n}\right)$, endowed with the bilinear pairing defined by the intersection product, is a hyperbolic lattice. Let $C r_{n}$ be the subgroup of $\operatorname{Aut}\left(A\left(X_{n}\right)\right)$ generated by the symmetric group $S_{n} \hookrightarrow A u t\left(A\left(X_{n}\right)\right)$ (acting on the last $n$ components) and the reflection $R: A\left(X_{n}\right) \rightarrow A\left(X_{n}\right)$ defined by $R(x):=x+(x \cdot e) e$, where $e:=\left[L^{\mathcal{K}_{0}(n)}\right]-\left[E_{1}^{\mathcal{K}_{0}(n)}\right]-\left[E_{2}^{\mathcal{K}_{0}(n)}\right]-\left[E_{3}^{\mathcal{K}_{0}(n)}\right]$. The group $C r_{n}$ is called the Cremona group.

It is obvious that $C r_{n}$ acts on the set of (open) half-lines of $A\left(X_{n}\right)$ with origin at 0 (rays). Moreover it also acts on the set of nef classes in $A\left(X_{n}\right)$ (see [2]) and, if $D$ is a nef divisor on $X_{n}$ such that $D^{2}=0$ then $[D] \in \partial \overline{N E}\left(X_{n}\right)$. These facts show that each one 
of the infinite rays $\eta \subseteq \partial \overline{N E}\left(X_{n}\right) \cap \partial Q\left(X_{n}\right) \cap\left[K_{X_{n}}\right]_{>0}$ provided by Theorem 11 gives rise to infinitely many rays contained in the same set: those belonging to the orbit of $\eta$ by the action of the Cremona group. Taking this into account, the last result of the paper completes the one provided by Theorem 1 .

Theorem 2. Let $n \geq 37$ be an integer and set $X_{n}$ and $S(n)$ as in the statement of Theorem 1. Let $\mathcal{R}$ be the set of rays in $A\left(X_{n}\right)$ of the form $\mathbb{R}_{>0}\left[D_{d, \alpha}\right]$ such that $d \in S(n) \backslash\{2,3,4\}$ and $\alpha \in E^{d}$. Then, for each ray $\eta \in \mathcal{R}$, the intersection of $\mathcal{R}$ with the orbit of $\eta$ by the action of $C r_{n}$ is finite. In particular, infinitely many of the rays in $\partial \overline{N E}\left(X_{n}\right) \cap \partial Q\left(X_{n}\right) \cap\left[K_{X_{n}}\right]_{>0}$ provided in Theorem 11 are generated by elements which belong to different orbits of the action of the Cremona group.

Proof. Given a ray $\eta$ in $A\left(X_{n}\right)$ we shall denote by $\eta^{\prime}$ to its primitive generator, that is, the generator $a_{0}\left[L^{\mathcal{K}_{0}(n)}\right]+\sum_{i=1}^{n} a_{i}\left[E_{i}^{\mathcal{K}_{0}(n)}\right]$ such that $\operatorname{gcd}\left(a_{0}, a_{1}, \ldots, a_{n}\right)=1$. Since the canonical class is fixed by the action of $C r_{n}$, it is clear that the result follows if we are able to prove that, for each positive integer $k$, the set $\left\{\left[K_{X_{n}}\right] \cdot \eta^{\prime} \leq k \mid \eta \in \mathcal{R}\right\}$ is finite. Let us see this fact.

Choose a positive integer $k$ and, reasoning by contradiction, assume that the above set is not finite. Let $\eta$ be an arbitrary ray in $\mathcal{R}$, say generated by $\left[D_{d, \alpha}\right]=m_{0}\left[L^{\mathcal{K}_{0}(n)}\right]-$ $\sum_{i=1}^{n} m_{i}\left[E_{i}^{\mathcal{K}_{0}(n)}\right]$. Set $\eta^{\prime}=m_{0}^{\prime}\left[L^{\mathcal{K}_{0}(n)}\right]-\sum_{i=1}^{n} m_{i}^{\prime}\left[E_{i}^{\mathcal{K}_{0}(n)}\right]$. Since the set of possible values of $m_{0}^{\prime}$ is unbounded and the set

$$
\left\{\frac{\left[K_{X_{n}}\right] \cdot \eta^{\prime}}{m_{0}^{\prime}} \leq \frac{k}{m_{0}^{\prime}} \mid \eta \in \mathcal{R}\right\}
$$

is not finite, one has that $\frac{\left[K_{X_{n}}\right] \cdot \eta^{\prime}}{m_{0}^{\prime}}<1$ for some $\eta \in \mathcal{R}$. But

$$
\frac{\left[K_{X_{n}}\right] \cdot \eta^{\prime}}{m_{0}^{\prime}}=\frac{\left[K_{X_{n}}\right] \cdot\left[D_{d, \alpha}\right]}{m_{0}}=\frac{K_{X^{d}} \cdot D_{d, \alpha}}{m_{0}} \geq 1,
$$

where the last inequality holds by the proof of Lemma 3 in [26]. This is a contradiction.

\section{REFERENCES}

[1] J. Alexander, A. Hirschowitz, Polynomial interpolation in several variables, J. Algebraic Geom. 4 (1995), 201-222.

[2] P. Biran, Constructing new ample divisors out of old ones, Duke Math. J. 98, (1999), no.1, 113-135.

[3] M. Brunella, Birational Geometry of Foliations, IMPA (2000).

[4] C. Ciliberto, R. Miranda, Degenerations of planar linear systems, J. reine angew. Math. 501 (1998), $191-220$.

[5] C. Ciliberto, R. Miranda, Linear systems of plane curves with base points of equal multiplicity, Trans. Amer. Math. Soc. 352 (2000), no. 9, 4037-4050.

[6] T. de Fernex, Negative curves on very general blow-ups of $\mathbb{P}^{2}$. Projective varieties with unexpected properties, 199-207, Walter de Gruyter GmbH \& Co. KG, Berlin, 2005.

[7] I. Dolgachev, D. Ortland, Point sets in projective spaces and theta functions, Astérisque, 165, 1988.

[8] M. Dumnicki, W. Jarnicki, New effective bounds on the dimension of a linear system in $\mathbb{P}^{2}$, J. Symbolic Comput. 42 (2007), no. 6, 621-635.

[9] P. du Val, On the Kantor group of a set of points in a plane, Proc. London Math. Soc. 42 (1937), no. $2,18-51$.

[10] L. Évain, La fonction de Hilbert de la réunion de $4^{h}$ gros points génériques de $\mathbb{P}^{2}$ de même multiplicité, J. Algebraic Geom. 8 (1999), no. 4, 787-796.

[11] C. Galindo, F. Monserrat, The cone of curves associated to a plane configuration, Comment. Math. Helv. 80 (2005), $75-93$

[12] C. Galindo, F. Monserrat, Algebraic integrability of foliations of the plane, J. Diff. Equations 231 (2006), no. $2,611-632$. 
[13] C. Galindo, F. Monserrat, On the characterization of algebraically integrable plane foliations, to appear in Trans. Amer. Math. Soc.

[14] J. García de la Fuente, Geometría de los sistemas lineales de series de potencias en dos variables, $\mathrm{Ph}$. D. thesis, Valladolid University, 1989, (in Spanish).

[15] A. Gimigliano, On linear systems of plane curves, Thesis, Queen's University, Kingston (1987).

[16] X. Gómez-Mont and L. Ortiz, Sistemas dinámicos holomorfos en superficies, Aportaciones Matemáticas 3, Sociedad Matemtica Mexicana, 1989 (in Spanish).

[17] B. Harbourne, The geometry of rational surfaces and Hilbert functions of points in the plane, Can. Math. Soc. Conf. Proc. 6 (1986), 95-111.

[18] B. Harbourne, Iterated blow-ups and moduli for rational surfaces, Algebraic geometry (Sundance, UT, 1986), 101-117, Lecture Notes in Math. 1311, Springer, Berlin (1988).

[19] B. Harbourne, Points in good position in $\mathbb{P}^{2}$, Zero-dimensional schemes (Ravello, 1992), 213-229, Walter de Gruyter, Berlin (1994).

[20] B. Harbourne, Rational surfaces with $K^{2}>0$, Proc. Amer. Math. Soc. 124 (1996), 727-733.

[21] R. Hartshorne, Algebraic geometry, GTM 52, Springer-Verlag, 1987.

[22] A. Hirschowitz, Une conjecture pour la cohomologie des diviseurs sur les surfaces rationnelles génériques, J. reine angew. Math. 397 (1989), 208-213.

[23] S. Kleiman, The enumerative theory of singularities, Real and Complex Singularities, Oslo 1976 (P. Holm, ed.), Sijthoff \& Noordhoof (1977), 297-396.

[24] S. Kleiman, Multiple point formulas I: Iteration, Acta Math. 147 (1981), 13-49.

[25] J. Kollár, Rational curves on algebraic varieties, Ergeb. Math. Grenzgeb. (3) 32, Springer-Verlag, 1996.

[26] A. Lins Neto, Some examples for the Poincaré and Painlevé problems, Ann. Sc. Éc. Norm. Sup. 35 (2002), 231-266.

[27] D. McDuff, L. Polterovich, Symplectic packings and algebraic geometry, with an appendix by Yael Karshon, Invent. Math. 115 (1994), 405-434.

[28] F. Monserrat, Curves having one place at infinity and linear systems on rational surfaces, J. Pure Appl. Algebra 211 (2007), 685-701.

[29] M. Nagata, on the 14-th problem of Hilbert, Amer. J. Math. 81 (1959), 766-772.

[30] M. Nagata, On rational surfaces II, Mem. Coll. Scil. Univ. Kyoto 33 (1960), 271-293.

[31] H. Poincaré, Sur l'intégration algébrique des équations différentielles du premier ordre et du premier degré (I), Rend. Circ. Mat. Palermo 5 (1891), 161-191.

[32] H. Poincaré, Sur l'intégration algébrique des équations différentielles du premier ordre et du premier degré (II), Rend. Circ. Mat. Palermo 11 (1897), 193-239.

[33] B. Segre, Alcune questioni su insiemi finiti di punti in geometria algebrica, Atti Convegno Intern. di Geom. Alg. di Torino (1961), 15-33.

[34] A. Seidenberg, Reduction of singularities of the differentiable equation $A d y=B d x$, Amer. J. Math. 90 (1968), 248-269.

[35] S. Yang, Linear systems of plane curves with base points of bounded multiplicity, J. Algebraic Geom. 16 (2007), no. 1, 19-38.

Departamento de Matemática Aplicada, Universidad Politécnica de Valencia, Camino de Vera s/n, 46022 VALencia, Spain

E-mail address: framonde@mat.upv.es 